\title{
nPDF constraints from the Large Hadron Electron Collider
}

\author{
Ilkka Helenius ${ }^{* a}$, Hannu Paukkunen ${ }^{b c d}$ and Nestor Armesto ${ }^{d}$ \\ ${ }^{a}$ Department of Astronomy and Theoretical Physics, Lund University, Sölvegatan 14A, SE-223 62 \\ Lund, Sweden \\ ${ }^{b}$ University of Jyvaskyla, Department of Physics, P.O. Box 35, FI-40014 University of Jyvaskyla, \\ Finland \\ ${ }^{c}$ Helsinki Institute of Physics, P.O. Box 64, FI-00014 University of Helsinki, Finland \\ ${ }^{d}$ IGFAE, Universidade de Santiago de Compostela, E-15782 Galicia, Spain \\ E-mail: ilkka.helenius@thep.lu.se, hannu.t.paukkunen@jyu.fi, \\ nestor.armestodusc.es
}

An updated analysis regarding the expected nuclear PDF constraints from the future Large Hadron Electron Collider ( $\mathrm{LHeC}$ ) experiment is presented. The new study is based on a more flexible small- $x$ parametrization which provides less biased uncertainty estimates in the region where there are currently no data constraints. The effect of the $\mathrm{LHeC}$ is quantified by directly including a sample of pseudodata according to the expected precision of this planned experiment. As a result, a significant reduction of the small- $x$ uncertainties in sea quarks and gluons is observed.

XXIV International Workshop on Deep-Inelastic Scattering and Related Subjects

11-15 April, 2016

DESY Hamburg, Germany

\footnotetext{
* Speaker.
} 


\section{Introduction}

The Large Hadron Electron Collider ( $\mathrm{LHeC}$ ) project consists on the construction of a new electron accelerator providing an electron beam that would collide with the hadron beam from the Large Hadron Collider (LHC). The design also allows a synchronous operation of e-p collisions at the $\mathrm{LHeC}$ and p-p collisions at the LHC, as well as replacing the proton beam with heavy ions. A detailed description about the design can be found in the conceptual design report [1].

Accurately determined parton distribution functions (PDFs) are essential to all perturbative QCD (pQCD) based theory predictions. This holds also for nuclear collisions, where the nuclear modifications of the PDFs are needed to obtain a precise $\mathrm{pQCD}$ baseline for heavy-ion physics at the LHC, and also for future high-energy colliders, such as the Future Circular Collider (FCC) [2]. In this talk we focus on the current status of the nuclear PDFs (nPDFs), and quantify the potential of $\mathrm{e}-\mathrm{Pb}$ collisions at the $\mathrm{LHeC}$ to improve the accuracy of the future nPDF analyses. Other interesting e- $A$ physics are described e.g. in Refs. [1,3].

\section{New baseline nPDF fit}

The nPDFs can be determined via a similar global analysis as the free proton PDFs. The scale evolution is given by the DGLAP equations, but a non-perturbative input function $f\left(x, Q_{0}^{2}\right)$ at the initial scale $Q_{0}^{2}$ of the fit is required for the $x$ dependence. The ansatz for $f\left(x, Q_{0}^{2}\right)$ should include enough freedom to capture all the relevant features of data used in the analysis but, on the other hand, keep the number of parameters reasonable to ensure the convergence of the fit and not to be sensitive to the fluctuations of the individual data points.

The data used in the current fits comes from deep inelastic scattering (DIS), Drell-Yan dilepton production and inclusive pion production in d-Au collisions at RHIC. Since most of the data are from fixed-target experiments, the limited collision energy restricts the kinematic reach of the data, as discussed e.g. in Ref. [3]. Therefore, the current fits NCTEQ15 [4], KA15 [5], DSSZ [6], EPS09 [7] and HKN07 [8] include data that are restricted to $x \gtrsim 5 \cdot 10^{-3}$ and $Q^{2} \lesssim 100 \mathrm{GeV}^{2}$. The recent dijet, charged hadron and $W^{ \pm} / Z$ data from $\mathrm{p}-\mathrm{Pb}$ collisions at the LHC provide some additional constraints for the nPDF analyses [9], but in practice do not increase the small- $x$ reach. With the $\mathrm{LHeC}$, the kinematic reach of e-A DIS cross section could be extended by more than three orders of magnitude in both $x$ and $Q^{2}$ providing a kinematic reach comparable to the current proton PDF fits.

The nPDF uncertainties are usually quantified via the Hessian method [10], which allows a simple way to study how the uncertainties propagate into physical observables. Within this method the parameters (in a diagonalized basis) are allowed to vary within some $\Delta \chi^{2}$ value (here, $\Delta \chi^{2}=$ 17). The uncertainty estimates are always tied to the functional form of $f\left(x, Q_{0}^{2}\right)$, especially in the region where there are no guidance from the data. In the EPS09 analysis [7] the ratios $R$ of parton densities in a bound proton inside a nucleus over that in a free proton were parametrized with a piecewise function,

$$
R^{\mathrm{EPS} 09}(x)=\left\{\begin{array}{lc}
a_{0}+\left(a_{1}+a_{2} x\right)\left(e^{-x}-e^{-x_{a}}\right), & x \leq x_{a} \\
b_{0}+b_{1} x+b_{2} x^{2}+b_{3} x^{3}, & x_{a} \leq x \leq x_{e} \\
c_{0}+\left(c_{1}-c_{2} x\right)(1-x)^{-\beta}, & x_{e} \leq x \leq 1
\end{array}\right.
$$


This functional form does not allow much freedom for the small- $x$ behaviour, as shown in the lefthand panel of Figure 1 - the function at small $x$ is monotonic. Theoretically, this kind of behaviour appears reasonable but since global fits should rather indicate the uncertainty originating from the used data, a more flexible form should be used to study the realistic potential of the LHeC. The small- $x$ form used in this study is

$$
R\left(x \leq x_{a}\right)=a_{0}+a_{1}\left(x-x_{a}\right)^{2}+\sqrt{x}\left(x_{a}-x\right)\left[a_{2} \log \left(\frac{x}{x_{a}}\right)+a_{3} \log ^{2}\left(\frac{x}{x_{a}}\right)+a_{4} \log ^{3}\left(\frac{x}{x_{a}}\right)\right],
$$

which provides much more freedom for the small- $x$ behaviour reducing the bias due to the ansatz significantly. This is illustrated in the right-hand panel of Figure 1, showing the $R\left(x<x_{a}, Q_{0}^{2}\right)$ with a few different possible parameter values.
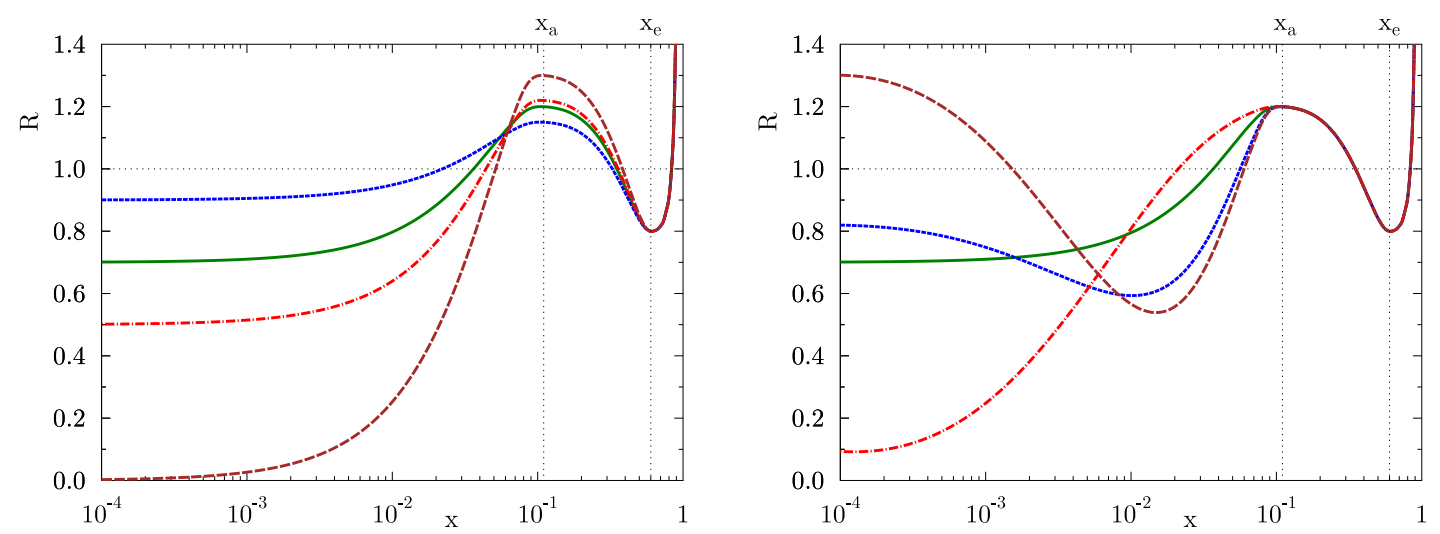

Figure 1: The fit function behaviour from the EPS09-style ansatz (left) and from the new ansatz with more freedom at small values of $x$ used in this study (right).

To have a more realistic estimate for the current uncertainties, a new baseline fit was performed. The setup for the baseline fit was very similar as in the EPS09 analysis, but now including the more flexible form from Eq. (2.2) at low $x$. The new baseline fit reflects the lack of control at small values of $x$ from the data by providing vastly larger uncertainties than the original EPS09 analysis, as shown in Figure 2, even though the data constraints are the same.

\section{Fit to pseudodata}

The impact of the $\mathrm{LHeC}$ pseudodata on the $\mathrm{nPDF}$ uncertainties was studied by performing a new nPDF analysis using pseudodata generated according to $\mathrm{LHeC}$ expectations, but otherwise keeping the details as in the baseline fit. The pseudodata were generated for neutral current (NC) DIS assuming per-nucleon luminosities $\mathscr{L}_{\mathrm{ep}}=10 \mathrm{fb}$ and $\mathscr{L}_{\mathrm{ePb}}=1 \mathrm{fb}$. The kinematical window studied was $10^{-5}<x<1$ and $2<Q^{2}<10^{5} \mathrm{GeV}^{2}$ and the data were based on the EPS09 nPDFs, including also realistic fluctuations with the cited luminosities [11]. A comparison between the baseline fit and the generated pseudodata is shown in Figure 3 displaying ratios of reduced cross sections between e- $\mathrm{Pb}$ and e-p collisions. Figure 3 shows also the corresponding comparison after including the pseudodata into the fit. A drastic reduction of the nPDF uncertainties is observed once the pseudodata are included to the analysis. 



Figure 2: The nPDF modifications for valence (left) and sea (middle) quarks, and gluons (right) at $Q^{2}=1.69 \mathrm{GeV}^{2}$ (upper panels) and $Q^{2}=10 \mathrm{GeV}^{2}$ (lower panels). The dashed lines show the uncertainty according to the EPS09 analysis and the colour band the result of the new baseline fit with the more flexible low- $x$ parametrization.

To study the improvements in more detail, Figure 4 compares the nPDF uncertainties for valence quarks, sea quarks and gluons from the baseline fit and from the new fit including also the pseudodata. The large- $x$ valence quarks are already well constrained by the current data but the improvement is significant for the small- $x$ sea quarks and gluons. Improvement like this would substantially solidify the pQCD baseline for the heavy-ion physics at the LHC, and also at the FCC, already from $p_{T} \sim 3 \mathrm{GeV} / \mathrm{c}$ on.

\section{Summary}

The impact of the NC DIS data from the LHeC to the nPDFs has been studied. To obtain a more realistic estimates than the present nPDF uncertainties, a new baseline fit was performed applying a more flexible form for the small- $x$ behaviour that is included in the current fits. Due to the new parametrization, the baseline analysis possesses significantly larger small- $x$ uncertainties than the current public analyses. A set of pseudodata was generated according to the expected LHeC specifications, and a new fit including the pseudodata was performed and the uncertainties were compared to the baseline fit without the pseudodata. The results show a substantial reduction of small- $x$ uncertainties for sea quarks and gluons, improving the accuracy of pQCD baseline for heavy-ion collisions in the kinematic region relevant for the LHC and the FCC.

\section{Acknowledgments}

This research was supported by the European Research Council grant HotLHC ERC-2011- 

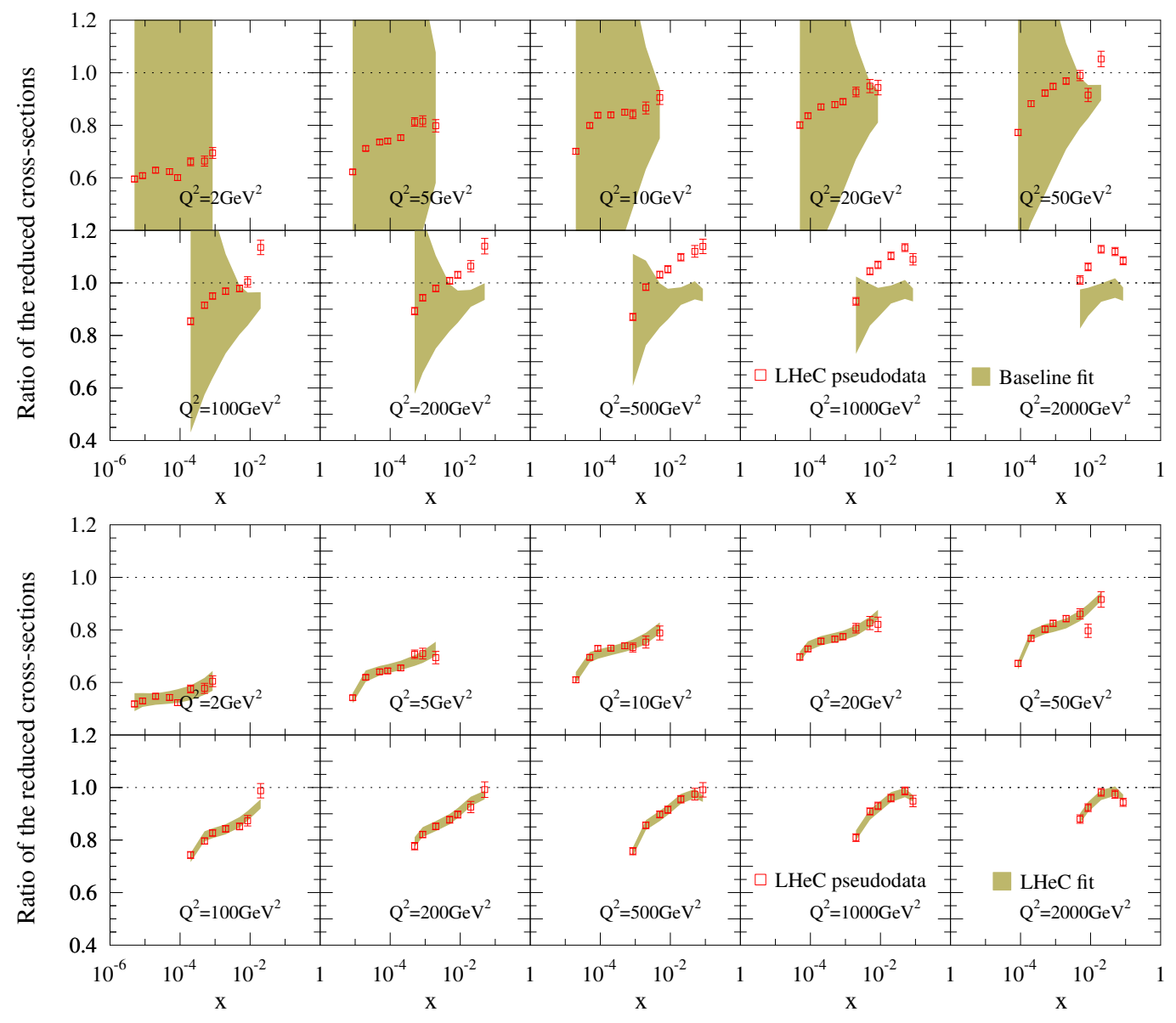

Figure 3: Ratios of the reduced NC DIS cross section between e-Pb and e-p collisions. The generated pseudodata are shown with red markers and compared to the baseline fit (upper panels) and to a new fit using the $\mathrm{LHeC}$ pseudodata (lower panels). The fit uncertainties are shown with yellowish colour bands.

StG-279579 and by Xunta de Galicia (Conselleria de Educacion) - H. P. and N. A. are part of the Strategic Unit AGRUP2015/11. N. A. is supported by the People Programme (Marie Curie Actions) of the European Union Seventh Framework Programme FP7/2007-2013/ under REA grant agreement \#318921 and Ministerio de Ciencia e Innovaciónn of Spain under project FPA2014-58293C2-1-P. I .H. has been supported by the MCnetITN FP7 Marie Curie Initial Training Network, contract PITN-GA-2012-315877 and has received funding from the European Research Council (ERC) under the European Union Horizon 2020 research and innovation programme (grant agreement No 668679).

\section{References}

[1] LHeC Study Group collaboration, J. L. Abelleira Fernandez et al., A Large Hadron Electron Collider at CERN: Report on the Physics and Design Concepts for Machine and Detector, J. Phys. G39 (2012) 075001, [arXiv:1206.2913].

[2] A. Dainese et al., Heavy ions at the Future Circular Collider, arXiv:1605.01389. 


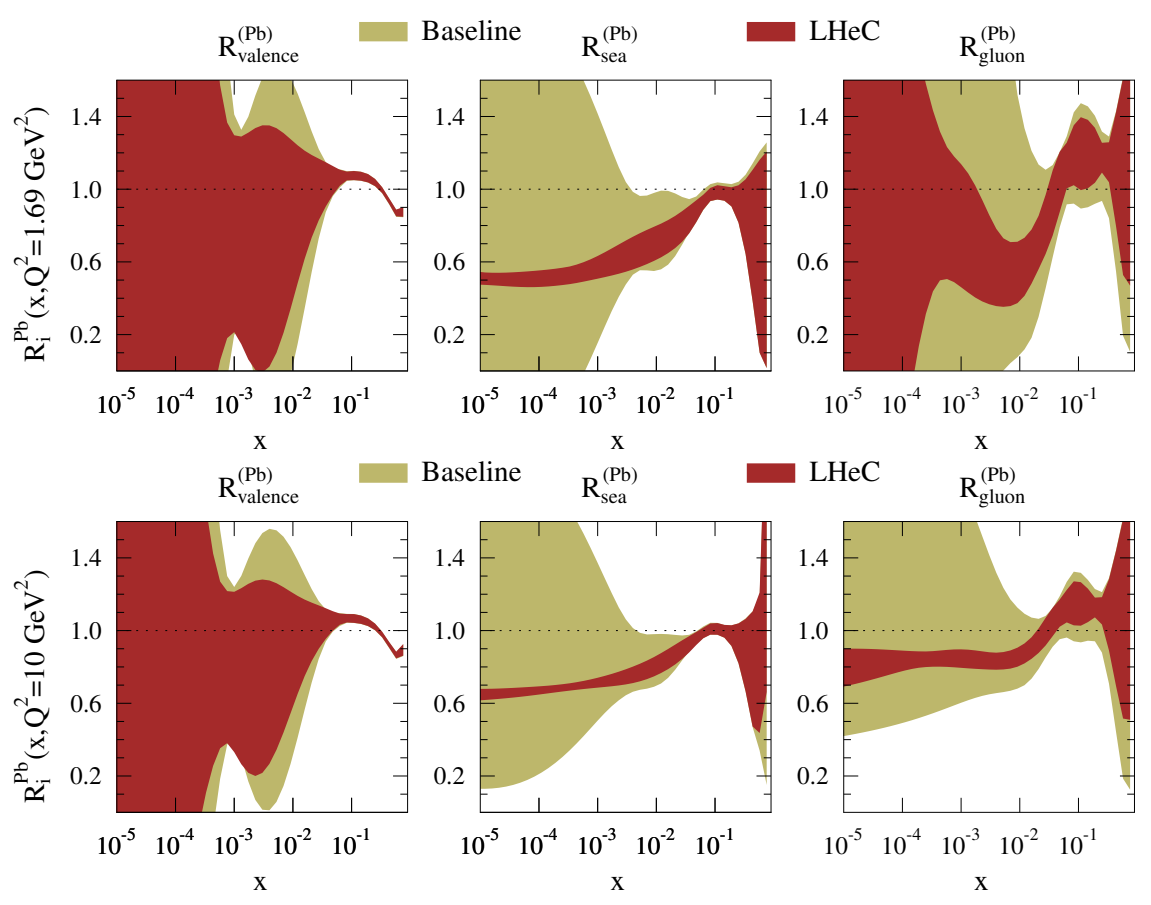

Figure 4: The nuclear modification of the PDFs for valence quarks (left), sea quarks (middle) and gluons (right) for two scales $Q^{2}=1.69 \mathrm{GeV}^{2}$ (upper panels) and $Q^{2}=10 \mathrm{GeV}^{2}$ (lower panels). The baseline fit is shown as yellowish colour bands and the fit including also $\mathrm{LHeC}$ pseudodata as dark red bands.

[3] I. Helenius, H. Paukkunen and N. Armesto, Electron-Ion Physics with the LHeC, PoS DIS2015 (2015) 226, [arXiv:1509.02802].

[4] K. Kovarik et al., nCTEQ15 - Global analysis of nuclear parton distributions with uncertainties in the CTEQ framework, Phys. Rev. D93 (2016) 085037, [arXiv:1509.00792].

[5] H. Khanpour and S. Atashbar Tehrani, Global Analysis of Nuclear Parton Distribution Functions and Their Uncertainties at Next-to-Next-to-Leading Order, Phys. Rev. D93 (2016) 014026, [arXiv:1601.00939].

[6] D. de Florian, R. Sassot, P. Zurita and M. Stratmann, Global Analysis of Nuclear Parton Distributions, Phys. Rev. D85 (2012) 074028, [arXiv:1112.6324].

[7] K. J. Eskola, H. Paukkunen and C. A. Salgado, EPS09: A New Generation of NLO and LO Nuclear Parton Distribution Functions, JHEP 04 (2009) 065, [arXiv: 0902 . 4154].

[8] M. Hirai, S. Kumano and T. H. Nagai, Determination of nuclear parton distribution functions and their uncertainties in next-to-leading order, Phys. Rev. C76 (2007) 065207, [arXiv: 0709.3038 ].

[9] N. Armesto, H. Paukkunen, J. M. Penín, C. A. Salgado and P. Zurita, An analysis of the impact of LHC Run I proton-lead data on nuclear parton densities, Eur. Phys. J. C76 (2016) 218, [arXiv:1512.01528].

[10] J. Pumplin, D. Stump, R. Brock, D. Casey, J. Huston, J. Kalk et al., Uncertainties of predictions from parton distribution functions. 2. The Hessian method, Phys. Rev. D65 (2001) 014013, [hep-ph/0101032].

[11] M. Klein. Private communication. 